Nonlinear response of a forced van der Pol-Duffing oscillator at non-resonant bifurcations of codimension two

\author{
This paper was published in Chaos, Solitons \& Fractals
}

Vol 41, No.3, pp.1467-1475, August 2009.

J.C. Ji, N. Zhang

Faculty of Engineering

University of Technology, Sydney

PO Box 123, Broadway, NSW 2007, Australia

Email: jinchen.ji@eng.uts.edu.au

Total number of pages: $17+4=21$

Total number of figures: 3 


\title{
Nonlinear response of a forced van der Pol-Duffing oscillator at non-resonant bifurcations of codimension two
}

\begin{abstract}
Non-resonant bifurcations of codimension two may appear in the controlled van der PolDuffing oscillator when two critical time delays corresponding to a double Hopf bifurcation have the same value. With the aid of centre manifold theorem and the method of multiple scales, the non-resonant response and two types of primary resonances of the forced van der Pol-Duffing oscillator at non-resonant bifurcations of codimension two are investigated by studying the possible solutions and their stability of the four-dimensional ordinary differential equations on the centre manifold. It is shown that the non-resonant response of the forced oscillator may exhibit quasi-periodic motions on a two- or threedimensional (2D or 3D) torus. The primary resonant responses admit single and mixed solutions and may exhibit periodic motions or quasi-periodic motions on a 2D torus. Illustrative examples are presented to interpret the dynamics of the controlled system in terms of two dummy unfolding parameters and exemplify the periodic and quasi-periodic motions. The analytical predictions are found to be in good agreement with the results of numerical integration of the original delay differential equation.
\end{abstract}

Keywords: van der Pol-Duffing oscillator, nonlinear feedback control, time delay, codimension two bifurcation, non-resonant Hopf bifurcations, primary resonances. 


\section{Introduction}

Stability and dynamics of van der Pol-Duffing oscillator (or harmonic oscillators) under delayed feedback control have received considerable interest [1-5]. Presence of time delays in the feedback control may induce instability and complex behaviour of the controlled system.

An externally forced van der Pol-Duffing oscillator under a linear-plus-nonlinear feedback control considered in the present paper is of the form

$$
\begin{aligned}
& \ddot{x}-\left(\mu-\beta x^{2}\right) \dot{x}+\omega^{2} x+\alpha x^{3}=e_{0} \cos \left(\Omega_{0} t\right)+p x(t-\tau)+q \dot{x}(t-\tau)+k_{1} x^{3}(t-\tau) \\
& +k_{2} \dot{x}^{3}(t-\tau)+k_{3} \dot{x}(t-\tau) x^{2}(t-\tau)+k_{4} \dot{x}^{2}(t-\tau) x(t-\tau)
\end{aligned}
$$

where an overdot indicates the differentiation with respect to time $t, x$ is the displacement, $\omega$ is the natural frequency, $\alpha$ is the coefficient of the nonlinear term, $\mu>0, \beta>0, e_{0}$ and $\Omega_{0}$ represent the amplitude and frequency of the external excitation, $p$ and $q$ are the proportional and derivative linear feedback gains, $k_{i}(i=1,2,3,4)$ are the weakly nonlinear feedback gains, and $\tau$ denotes the time delay occurring in the feedback path. The corresponding autonomous system (obtained by letting $e_{0}=0$ in equation (1)) is given by

$$
\begin{gathered}
\ddot{x}-\mu \dot{x}+\omega^{2} x-p x(t-\tau)-q \dot{x}(t-\tau)+\beta x^{2} \dot{x}+\alpha x^{3}-k_{1} x^{3}(t-\tau)-k_{2} \dot{x}^{3}(t-\tau) \\
-k_{3} \dot{x}(t-\tau) x^{2}(t-\tau)-k_{4} \dot{x}^{2}(t-\tau) x(t-\tau)=0 .
\end{gathered}
$$

It was shown that the trivial equilibrium of the autonomous system (2) may lose its stability via a subcritical or a supercritical Hopf bifurcation and regain its stability via a reverse subcritical or a supercritical Hopf bifurcation as the time delay increases [6]. It was found that an interaction of two Hopf bifurcations may occur when the two critical time delays corresponding to a double Hopf bifurcation have the same value. In the vicinity of non-resonant Hopf bifurcations, the autonomous system (2) was found to have the initial equilibrium solution, two periodic solutions and a quasi-periodic solution on 2D 
torus [7]. The primary objective of the present paper is to study the non-resonant response and primary resonance response of the controlled system (1) that results from an interaction of the external excitation and the bifurcation solutions of the corresponding autonomous system at non-resonant Hopf bifurcations of codimension two.

The remainder of the present paper proceeds as follows. In the next section, the existence of non-resonant Hopf bifurcations is briefly reviewed for the autonomous system. The reduction of the delay differential equation is concisely discussed in Section 3. The non-resonant response is numerically and analytically studied in Section 4. Two types of primary resonance responses of the controlled system are analytically studied in Sections 5 and 6 using the method of multiple time scales. In Section 7, illustrative examples are given to show the primary resonance response of the controlled system. Results are presented in Section 8.

\section{Existence of non-resonant Hopf bifurcations}

This section briefly reviews background materials on the existence of non-resonant bifurcations of codimension two of the corresponding autonomous system after the trivial equilibrium loses it stability. More details can be found in reference [7].

The characteristic equation for studying the stability of the trivial equilibrium of equation (2) is given by

$$
\lambda^{2}-\mu \lambda+\omega^{2}-p e^{-\lambda \tau}-q \lambda e^{-\lambda \tau}=0 .
$$

It is found that if $\left(q^{2}-\mu^{2}+2 \omega^{2}\right)>0, \quad\left(\mu^{2}-q^{2}\right)\left(q^{2}-\mu^{2}+4 \omega^{2}\right)<4 p^{2}<4 \omega^{4}$, equation (3) has two pairs of purely imaginary solutions given by $\lambda_{ \pm}= \pm \mathrm{i} \delta_{ \pm}$, with

$$
\delta_{ \pm}^{2}=\left(q^{2}-\mu^{2}+2 \omega^{2} \pm \sqrt{4\left(p^{2}-\omega^{4}\right)+\left(2 \omega^{2}+q^{2}-\mu^{2}\right)^{2}}\right) / 2,
$$

where $\delta_{+}>\delta_{-}>0$. 
Two sets of the critical time delays corresponding to two pairs of purely imaginary eigenvalues are given by

$$
\tau_{1 \mathrm{c}, \mathrm{n}}=\left(s_{1}+2 n \pi\right) / \delta_{+}, \quad \tau_{2 \mathrm{c}, \mathrm{n}}=\left(s_{2}+2 n \pi\right) / \delta_{-}, \quad n=0,1,2 \ldots
$$

where $0 \leq s_{1}<2 \pi, 0 \leq s_{2}<2 \pi, \sin \left(s_{1,2}\right)=\left(\mu p+q \omega^{2}-q \delta_{ \pm}^{2}\right) \delta_{ \pm} /\left(p^{2}+q^{2} \delta_{ \pm}^{2}\right)$.

For clarity, the bifurcations occurring at points $\left(\delta_{+}, \tau_{1 \mathrm{c}, \mathrm{n}}\right)$ and $\left(\delta_{-}, \tau_{2 \mathrm{c}, \mathrm{n}}\right)$ will be termed the first and second Hopf bifurcation. The frequencies of the Hopf bifurcations, namely $\delta_{01}$ and $\delta_{02}$, are then given by $\delta_{01}=\delta_{+}, \delta_{02}=\delta_{-}$. An intersection of the first and second Hopf bifurcations may lead to a non-resonant or resonant Hopf-Hopf interaction, depending on the ratio of the frequencies of the Hopf bifurcations. Such an intersection is usually referred to as the point of codimension two bifurcations in the context of dynamical system theory [8]. Non-resonant Hopf bifurcations is considered in the present paper.

\section{Reduction of the centre manifold}

For brevity, it is assumed that an intersection of non-resonant Hopf bifurcations occurs at the point $\left(p_{0}, q_{0}, \tau_{0}\right)$, where equation (3) has two pairs of purely imaginary roots $\pm \mathrm{i} \delta_{01}$, $\pm \mathrm{i} \delta_{02}$, and all other roots have negative real parts. In order to study the dynamics of the oscillator in the neighbourhood of the point $\left(p_{0}, q_{0}, \tau_{0}\right)$, three small perturbation parameters, namely $\alpha_{1}, \alpha_{2}$ and $\alpha_{3}$, are introduced in equation (1) in terms of $p=p_{0}+\alpha_{1}$, $q=q_{0}+\alpha_{2}, \tau=\tau_{0}+\alpha_{3}$. These perturbation parameters can conveniently account for the small variations of the critical linear feedback gains and the critical time delay.

By following the normal procedure for the reduction of delay differential equations to ordinary differential equations [9-11] and treating the external excitation in equation (1) as an additional perturbation term, the four-dimensional ordinary differential equations governing the local flow on the centre manifold can be expressed in the component form as 


$$
\begin{aligned}
& \dot{z}_{1}=l_{11} z_{1}+\left(\delta_{1}+l_{12}\right) z_{2}+l_{13} z_{3}+l_{14} z_{4}+f_{10}\left(z_{1}, z_{2}, z_{3}, z_{4}\right)+e_{10} \cos (\Omega t), \\
& \dot{z}_{2}=\left(-\delta_{1}+l_{21}\right) z_{1}+l_{22} z_{2}+l_{23} z_{3}+l_{24} z_{4}+f_{20}\left(z_{1}, z_{2}, z_{3}, z_{4}\right)+e_{20} \cos (\Omega t), \\
& \dot{z}_{3}=l_{31} z_{1}+l_{32} z_{2}+l_{33} z_{3}+\left(\delta_{2}+l_{34}\right) z_{4}+f_{30}\left(z_{1}, z_{2}, z_{3}, z_{4}\right)+e_{30} \cos (\Omega t), \\
& \dot{z}_{4}=l_{41} z_{1}+l_{42} z_{2}+\left(-\delta_{2}+l_{43}\right) z_{3}+l_{44} z_{4}+f_{40}\left(z_{1}, z_{2}, z_{3}, z_{4}\right)+e_{40} \cos (\Omega t),
\end{aligned}
$$

where $\delta_{1}$ and $\delta_{2}$ are the normalized frequencies of Hopf bifurcations which are rescaled in the units of the critical time delay, $e_{10}=b_{12} e_{0}, e_{20}=b_{22} e_{0}, e_{30}=b_{32} e_{0}, e_{40}=b_{42} e_{0}$, the other coefficients and polynomial functions of order three $f_{i 0}\left(z_{1}, z_{2}, z_{3}, z_{4}\right)$ (where $i=1,2,3,4)$ are explicitly given in Section 3 of reference [7].

Depending on the relationship of two frequencies $\delta_{1}$ and $\delta_{2}$ with the forcing frequency $\Omega$, the nonlinear system (6) may exhibit non-resonant responses, primary, sub-harmonic and super-harmonic resonances, additive and difference resonances. The non-resonant response and primary resonance response of the system will be discussed in subsequent sections using the method of multiple scales [12], as the closed form of the solutions to equation (6) cannot be found analytically.

\section{Non-resonant response}

In this section, the dynamic behaviour of the system in the neighbourhood of the point of non-resonant bifurcations of codimension two will be studied based on a set of four averaged equations that determine the amplitudes and phases of the free oscillation terms contributing to the non-resonant response.

\subsection{First-order approximate solutions and their stability}

It is assumed that the approximate solutions to equation (6) in the neighbourhood of the trivial equilibrium are represented by an expansion of the form

$$
Z_{i}(t ; \varepsilon)=\varepsilon^{\frac{1}{2}} Z_{i 1}\left(T_{0}, T_{1}, \cdots\right)+\varepsilon^{\frac{3}{2}} Z_{i 2}\left(T_{0}, T_{1}, \cdots\right)+\cdots,(i=1,2,3,4) .
$$


where $\varepsilon$ is a non-dimensional small parameter, and the new multiple independent variables of time are introduced according to $T_{k}=\varepsilon^{k} t, k=0,1,2, \cdots$.

Substituting the approximate solution (7) into equation (6) and then balancing the like powers of $\varepsilon$ results in the following ordered perturbation equations

$$
\begin{array}{ll}
\varepsilon^{\frac{1}{2}}: & D_{0} z_{11}=\delta_{1} z_{21}+e_{1} \cos \left(\Omega T_{0}\right), \quad D_{0} z_{21}=-\delta_{1} z_{11}+e_{2} \cos \left(\Omega T_{0}\right), \\
D_{0} z_{31} & =\delta_{2} z_{41}+e_{3} \cos \left(\Omega T_{0}\right), \quad D_{0} z_{41}=-\delta_{2} z_{31}+e_{4} \cos \left(\Omega T_{0}\right), \\
\varepsilon^{\frac{3}{2}}: \quad D_{0} z_{12} & =g_{11}\left(z_{j 1}\right)+\delta_{1} z_{22}-D_{1} z_{11}+f_{11}\left(z_{j 1}\right), \\
D_{0} z_{22} & =g_{21}\left(z_{j 1}\right)-\delta_{1} z_{12}-D_{1} z_{21}+f_{21}\left(z_{j 1}\right), \\
D_{0} z_{32} & =g_{31}\left(z_{j 1}\right)+\delta_{2} z_{42}-D_{1} z_{31}+f_{31}\left(z_{j 1}\right), \\
D_{0} z_{42} & =g_{41}\left(z_{j 1}\right)-\delta_{2} z_{32}-D_{1} z_{41}+f_{41}\left(z_{j 1}\right),
\end{array}
$$

where $D_{0}=\partial / \partial T_{0}, D_{1}=\partial / \partial T_{1}, \quad g_{m 1}\left(z_{j 1}\right)=l_{m 1} z_{11}+l_{m 2} z_{21}+l_{m 3} z_{31}+l_{m 4} z_{41}, \quad(m=1,2,3,4)$, the coefficients $l_{i j}$ in equation (6) have been rescaled in terms of $l_{i j}=\varepsilon \bar{l}_{i j}$ and the overbars in $\bar{l}_{i j}$ have been removed for brevity. The amplitudes of the excitations in equation (6) have been rescaled in terms of $e_{10}=\varepsilon^{\frac{1}{2}} e_{1}, e_{20}=\varepsilon^{\frac{1}{2}} e_{2}, e_{30}=\varepsilon^{\frac{1}{2}} e_{3}, e_{40}=\varepsilon^{\frac{1}{2}} e_{4}$. The $f_{i 1}\left(z_{j 1}\right)$ are nonlinear functions of $z_{j 1}(j=1,2,3,4)$.

The solutions to equation (8) can be written in a general form as

$$
\begin{aligned}
& z_{11}=r_{1} \cos \left(\delta_{1} T_{0}+\phi_{1}\right)+A_{1} \cos \left(\Omega T_{0}\right)+A_{2} \sin \left(\Omega T_{0}\right), \\
& z_{21}=-r_{1} \sin \left(\delta_{1} T_{0}+\phi_{1}\right)+B_{1} \cos \left(\Omega T_{0}\right)+B_{2} \sin \left(\Omega T_{0}\right), \\
& z_{31}=r_{2} \cos \left(\delta_{2} T_{0}+\phi_{2}\right)+A_{3} \cos \left(\Omega T_{0}\right)+A_{4} \sin \left(\Omega T_{0}\right), \\
& z_{41}=-r_{2} \sin \left(\delta_{2} T_{0}+\phi_{2}\right)+B_{3} \cos \left(\Omega T_{0}\right)+B_{4} \sin \left(\Omega T_{0}\right),
\end{aligned}
$$

where $r_{1}, r_{2}, \phi_{1}$ and $\phi_{2}$ represent the amplitudes and phases of the free-oscillation terms, $A_{1}=\delta_{1} e_{2} /\left(\delta_{1}^{2}-\Omega^{2}\right), A_{2}=-\Omega e_{1} /\left(\delta_{1}^{2}-\Omega^{2}\right), B_{1}=\left(\Omega A_{2}-e\right) / \delta_{1}, B_{2}=-\Omega A_{1} / \delta_{1}$, 
$A_{3}=\delta_{2} e_{4} /\left(\delta_{2}^{2}-\Omega^{2}\right), A_{4}=-\Omega e_{3} /\left(\delta_{2}^{2}-\Omega^{2}\right), B_{3}=\left(\Omega A_{4}-e_{3}\right) / \delta_{2}, B_{4}=-\Omega A_{3} / \delta_{2}$.

It is noted in seeking the second-order solutions to equation (9) that, in addition to four secular terms that are proportional to $\sin \left(\delta_{1} t+\phi_{1}\right), \cos \left(\delta_{1} t+\phi_{1}\right), \sin \left(\delta_{2} t+\phi_{2}\right)$, and $\cos \left(\delta_{2} t+\phi_{2}\right)$, nearly secular terms may appear whenever the system possesses primary, secondary, additive or difference resonances. In particular, two types of primary resonances may occur when $\delta_{1} \cong \Omega$ or $\delta_{2} \cong \Omega$. Two types of sub-harmonic resonances happen when $\delta_{1} \cong \Omega / 3$ or $\delta_{2} \cong \Omega / 3$, and super-harmonic resonances take place when $\delta_{1} \cong 3 \Omega$ or $\delta_{2} \cong 3 \Omega$. It is also noted that for the nonlinear system given by equation (6) having $\delta_{1}>\delta_{2}$, a number of combinations of additive and difference resonances may appear. Specifically, additive resonances may appear when either $\delta_{1}+\delta_{2} \cong 2 \Omega$, or $2 \delta_{1}+\delta_{2} \cong \Omega$, or $\delta_{1}+2 \delta_{2} \cong \Omega$. Difference resonances may occur when either $\delta_{1}-\delta_{2} \cong 2 \Omega$, or $\delta_{1}-2 \delta_{2} \cong 2 \Omega$, or $2 \delta_{1}-\delta_{2} \cong \Omega$; or $2 \delta_{2}-\delta_{1} \cong \Omega$. As such, a total of 13 types of resonances may take place in the forced nonlinear response of the system. Thus in eliminating the terms that may produce secular terms in seeking the second-order approximate solutions, six cases need to be distinguished, namely, (a) primary resonances at either frequency of two Hopf bifurcations; (b) sub-harmonic resonances at either of two Hopf bifurcation frequencies; (c) super-harmonic resonances at either of two Hopf bifurcation frequencies; (d) additive resonances; (e) difference resonances; and (f) nonresonant response when $\Omega$ is well separated from the above-mentioned resonances.

The averaged equations that determine the amplitude and phases of the free-oscillation terms in equation (11) are given by

$$
\begin{aligned}
& \dot{r}_{1}=-\mu_{1} r_{1}+s_{11} r_{1}^{3}+s_{12} r_{1} r_{2}^{2}, \quad \dot{r}_{2}=-\mu_{2} r_{2}+s_{21} r_{1}^{2} r_{2}+s_{22} r_{2}^{3}, \\
& r_{1} \dot{\theta}_{1}=r_{1} \delta_{1}+r_{1} \rho_{1}+s_{31} r_{1}^{3}+s_{32} r_{1} r_{2}^{2}, \quad r_{2} \dot{\theta}_{2}=r_{2} \delta_{2}+r_{2} \rho_{2}+s_{41} r_{1}^{2} r_{2}+s_{42} r_{2}^{3},
\end{aligned}
$$


where $\theta_{1}=\delta_{1} T_{0}+\phi_{1}, \theta_{2}=\delta_{2} T_{0}+\phi_{2}$, a dot indicates the differentiation with respect to time $T_{0}$, and the coefficients are not produced here for brevity.

The fixed points are obtained by setting $\dot{r}_{1}=\dot{r}_{2}=0$ in the first two equations and the stability of the fixed points can be determined by studying the eigenvalues of the corresponding Jacobian matrix. It is easy to note that equation (11) admits four solutions, which will be referred to here as Solutions S1, S2, S3, and S4.

Solution S1 is given by $\left(r_{1}, r_{2}\right)=(0,0)$ and is stable if $\mu_{1}>0, \mu_{2}>0$.

Solution S2 is given by $\left(r_{1}, r_{2}\right)=\left(\sqrt{\mu_{1} / s_{11}}, 0\right)$ for $\mu_{1} / s_{11}>0$. It is stable under $\mu_{1}<0$, $s_{21} \mu_{1}<s_{11} \mu_{2}$.

Solution S3 is given by $\left(r_{1}, r_{2}\right)=\left(0, \sqrt{\mu_{2} / s_{22}}\right)$ for $\mu_{2} / s_{22}>0$. It is stable when $\mu_{2}<0$, $s_{12} \mu_{2}<s_{22} \mu_{1}$.

Solution S4 is a 3D torus solution given by $\left(r_{1}, r_{2}\right)=\left(\sqrt{\left(s_{12} \mu_{2}-s_{22} \mu_{1}\right) /\left(s_{12} s_{21}-s_{11} s_{22}\right)}, \sqrt{\left(s_{21} \mu_{1}-s_{11} \mu_{2}\right) /\left(s_{12} s_{21}-s_{11} s_{22}\right)}\right)$ for $\left(s_{12} \mu_{2}-s_{22} \mu_{1}\right) /\left(s_{12} s_{21}-s_{11} s_{22}\right)>0,\left(s_{21} \mu_{1}-s_{11} \mu_{2}\right) /\left(s_{12} s_{21}-s_{11} s_{22}\right)>0$. Solution $S 4$ is asymptotically stable if $s_{11} r_{1}^{2}<-s_{22} r_{2}^{2}, s_{12} s_{21}<s_{11} s_{22}$.

The non-resonant response of the nonlinear system given by equation (6) is periodic motion if a stable Solution S1 exists. When Solutions S2 and S3 exist, the non-resonant response may be quasi-periodic motion on a $2 \mathrm{D}$ torus if the involving frequencies are incommensurate. The non-resonant response corresponding to Solution S2 involves two frequencies being the forcing frequency $\Omega$ and the frequency resulting from the first Hopf bifurcation with $\omega_{H 1}=\delta_{1}+\rho_{1}+\mu_{1} s_{31} / s_{11}$. Two frequencies of the response relating to Solution S3 are the forcing frequency $\Omega$ and the frequency resulting from the second Hopf bifurcation being $\omega_{H 2}=\delta_{2}+\rho_{2}+\mu_{2} s_{42} / s_{22}$. The non-resonant response relating to 
solution S4 is a quasi-periodic motion on a 3D torus, which can be viewed as a motion by adding a third periodic motion to the 2D quasi-periodic motion of Solutions S2 or S3. The three frequencies involved are the forcing frequency $\Omega$ and two frequencies from Hopf bifurcation being $\omega_{H 1}=\delta_{1}+\rho_{1}+\left[\left(s_{21} s_{32}-s_{22} s_{31}\right) \mu_{1}+\left(s_{12} s_{31}-s_{11} s_{32}\right)\right] /\left(s_{12} s_{21}-s_{11} s_{22}\right)$, $\omega_{H 2}=\delta_{2}+\rho_{2}+\left[\left(s_{21} s_{42}-s_{22} s_{41}\right) \mu_{1}+\left(s_{12} s_{41}-s_{11} s_{42}\right) \mu_{2}\right] /\left(s_{12} s_{21}-s_{11} s_{22}\right)$.

It can be concluded that the non-resonant response of the controlled system at nonresonant Hopf bifurcations of codimension two may be periodic motion having frequency $\Omega$, quasi-periodic motions on a $2 \mathrm{D}$ torus having frequencies $\Omega$ and $\omega_{H 1}$ (or $\omega_{H_{2}}$ ), or quasi-periodic motions on a $3 \mathrm{D}$ torus having frequencies $\Omega, \omega_{H 1}$ and $\omega_{H 2}$.

\subsection{Illustrative example}

For a specific system with the parameters in equation (2) given by $\mu=0.1, \omega=1.0$, $p=-0.4, \quad \alpha=0.4, \quad \beta=0.5, k_{1}=0.2, k_{2}=k_{3}=0.0, k_{4}=0.5$, and $q=-0.402189$, as discussed in Section 2, two frequencies of non-resonant Hopf bifurcations of the corresponding autonomous system are found from equation (4) to be $\delta_{01}=1.28038$ and $\delta_{02}=0.71582$. The four solutions for this specific system can be easily found as follows:

Solution S1 being $r_{1}=r_{2}=0.0$;

Solution S2 being $r_{1}=2.92672 \sqrt{-0.743886 \alpha_{1}-0.22715 \alpha_{2}-0.19944 e_{0}^{2}}, r_{2}=0$;

Solution S3 being $r_{1}=0, \quad r_{2}=1.69862 \sqrt{-0.224145 \alpha_{1}-0.692885 \alpha_{2}-0.330278 e_{0}^{2}}$;

Solution S4 is given by $r_{1}=6.68465 \sqrt{-0.252459 \alpha_{1}-0.062161 \alpha_{2}-0.061225 e_{0}^{2}}$,

$$
r_{2}=1.49473 \sqrt{10.7289 \alpha_{1}+1.81818 \alpha_{2}+2.2456 e_{0}^{2}} \text {. }
$$

Here the third dummy parameter has been set $\alpha_{3}=0.0$. If let either $\alpha_{1}=0.0$ or $\alpha_{2}=0.0$ in equation (11), the perturbation parameter $\alpha_{3}$ will involve in the four solutions and thus 
the influence of time delay on the non-resonant response of the system can be easily studied in a similar procedure.

Careful check on the existence of solutions and their stability conditions indicates that the $\left(\alpha_{1}, \alpha_{2}, e_{0}\right)$ parameter space can be divided into four regions. The boundaries of these regions are defined by four planes, namely; $P_{1}, P_{2}, P_{3}$ and $P_{4}$, which are described by

$$
\begin{aligned}
& P_{1}: \alpha_{2}=-0.323495 \alpha_{1}-0.47667 e_{0}^{2}, \alpha_{1}>\left(-0.13598 e_{0}^{2}\right), \\
& P_{2}: \alpha_{2}=-3.27483 \alpha_{1}-0.87799 e_{0}^{2}, \alpha_{1}<\left(-0.13598 e_{0}^{2}\right), \\
& P_{3}: \alpha_{2}=-4.06137 \alpha_{1}-0.98494 e_{0}^{2}, \alpha_{1}>\left(-0.13598 e_{0}^{2}\right), \\
& P_{4}: \alpha_{2}=-5.90095 \alpha_{1}-1.23512 e_{0}^{2}, \alpha_{1}>\left(-0.13598 e_{0}^{2}\right) .
\end{aligned}
$$

Stable solution $\mathrm{S} 1$ exists in the region between surfaces $P_{1}$ and $P_{2}$. Crossing these two critical surfaces leads to solutions either S2 or S3, which bifurcates from solution S1 via appearance of a zero eigenvalue. Stable solution S2 exists in the region between planes $P_{2}$ and $P_{4}$, and stable solution S3 exists in the region between surfaces $P_{1}$ and $P_{3}$. Crossing the critical surface $P_{3}$, a secondary Hopf bifurcation solution with frequency $\omega_{\mathrm{H} 1}$ takes place from Solution S3, which leads to a 3D torus solution S4. Similarly, a secondary Hopf bifurcation solution with frequency $\omega_{\mathrm{H} 2}$ occurs from the solution S2 along surface $P_{4}$, giving rise to a 3D torus solution S4. The non-resonant response of the system (1) exhibits a periodic motion if stable solution S1 exists, quasi-periodic motions on 2D torus if either S2 or S3 stably exists, and a quasi-periodic motion on 3D torus if stable S4 exists.

The analytical predictions can be easily validated by numerical results as shown in Figure 1. Figure 1a shows the time history and phase portrait of a periodic solution for a combination of $\alpha_{1}=0.001, \alpha_{2}=0.1$ and $e_{0}=0.06$, which is located in the stable region for solution S1. A numerical solution for a quasi-periodic motion on a 2D torus (that is solution S3), as shown in Figure 1b, is obtained by choosing the perturbation parameters as 
$\alpha_{1}=0.001, \alpha_{2}=-0.0035$ and the amplitude of excitation $e_{0}=0.04$, which is in the region bounded by planes $P_{2}$ and $P_{3}$. Figure 1c shows the trajectory and phase portrait of a 3D torus motion corresponding to solution S4 for the parameters located in the region bounded by planes $P_{3}$ and $P_{4}$, which are given by $\alpha_{1}=0.001, \alpha_{2}=0.0053$, and $e_{0}=0.016$. It can be concluded that the analytical predictions of the response are in good agreement with the numerical results.

\section{Primary resonance at the frequency of the first Hopf bifurcation}

It is assumed that an approximate solution to the forced response of the system under primary resonances at the first Hopf bifurcation is of the general form that is given by equation (7). The frequency of external excitation is assumed to be almost equal to the linearized natural frequency according to $\delta_{1}=\Omega+\varepsilon \sigma_{1}$, where $\sigma_{1}$ is an external detuning parameter. The amplitudes of the excitations in equation (6) are rescaled in terms of $e_{10}=\varepsilon^{\frac{3}{2}} 2 e_{1}, e_{20}=\varepsilon^{\frac{3}{2}} 2 e_{2}, e_{30}=\varepsilon^{\frac{3}{2}} 2 e_{3}, e_{40}=\varepsilon^{\frac{3}{2}} 2 e_{4}$.

The first-order approximate solutions of the primary resonance response can be written in a general form as

$$
\begin{aligned}
& z_{11}=r_{1} \cos \left(\Omega T_{0}+\phi_{1}\right), \quad z_{21}=-r_{1} \sin \left(\Omega T_{0}+\phi_{1}\right) \\
& z_{31}=r_{2} \cos \left(\delta_{2} T_{0}+\phi_{2}\right), z_{41}=-r_{2} \sin \left(\delta_{2} T_{0}+\phi_{2}\right)
\end{aligned}
$$

where $r_{1}, r_{2}, \phi_{1}, \phi_{2}$ represent, respectively, the amplitudes and phases of the primary resonance response at the first Hopf bifurcation.

The averaged equations that determine the amplitudes and phases of the primary resonance response at the first Hopf bifurcation can be written as

$$
\begin{aligned}
& \dot{r}_{1}=-\mu_{1} r_{1}+s_{11} r_{1}^{3}+s_{12} r_{1} r_{2}^{2}+e_{1} \cos \phi_{1}-e_{2} \sin \phi_{1}, \\
& r_{1} \dot{\phi}_{1}=r_{1} \rho_{1}+s_{31} r_{1}^{3}+s_{32} r_{1} r_{2}^{2}-e_{1} \sin \phi_{1}-e_{2} \cos \phi_{1},
\end{aligned}
$$




$$
\begin{aligned}
& \dot{r}_{2}=-\mu_{2} r_{2}+s_{21} r_{1}^{2} r_{2}+s_{22} r_{2}^{3}, \\
& r_{2} \dot{\phi}_{2}=r_{2} \rho_{2}+s_{41} r_{1}^{2} r_{2}+s_{42} r_{2}^{3},
\end{aligned}
$$

where the coefficients are not produced here for brevity.

The steady state response of the primary resonance at the first Hopf bifurcation can be obtained by letting $\dot{r}_{1}=\dot{r}_{2}=0$ and $\dot{\phi}_{1}=0$ in equation (14). It is found that the amplitudes of the primary resonance response admits two types of solutions, which are referred to here as the single solutions with $r_{1} \neq 0$ and $r_{2}=0$, and the mixed solutions with $r_{1} \neq 0$ and $r_{2} \neq 0$.

For the single solutions, the so-called frequency-response equation is given by

$$
\left[\left(-\mu_{1}+s_{11} r_{1}^{2}\right)^{2}+\left(\rho_{1}+s_{31} r_{1}^{2}\right)^{2}\right] r_{1}^{2}=e_{1}^{2}+e_{2}^{2}
$$

The amplitudes of the single solutions can be numerically obtained from equation (15) for a given set of system parameters. The stability of the single solutions is determined by the eigenvalues of the corresponding Jacobian matrix of the first two equations of equation (14). The corresponding characteristic equation is given by

$$
\lambda^{2}+2 \mu_{e} \lambda+b_{e}=0
$$

where $\mu_{e}=\mu_{1}-2 s_{11} r_{1}^{2}, \quad b_{e}=\left(\rho_{1}+2 s_{31} r_{1}^{2}\right)^{2}+\left(-\mu_{1}+2 s_{11} r_{1}^{2}\right)^{2}-\left(s_{11}^{2}+s_{31}^{2}\right) r_{1}^{4}$. The single solution is asymptotically stable if both $\mu_{e}>0$ and $b_{e}>0$. The single solution may lose its stability via two different ways. The first case occurs when $\mu_{e}>0$ and $b_{e}=0$, for which a real eigenvalue passes through zero, i.e., $\lambda_{1}=0$. The second situation may happen when $\mu_{e}=0$ and $b_{e}>0$, for which a pair of complex eigenvalues cross the imaginary axis. The former will generate a saddle-node bifurcation while the latter a Neimark-Sacker bifurcation in the primary resonance response at the first Hopf bifurcation.

For the mixed solutions, the amplitudes of the mixed solutions are determined by two algebraic equations: 


$$
s_{21} r_{1}^{2}+s_{22} r_{2}^{2}-\mu_{2}=0, \quad c_{3} r_{2}^{6}+c_{2} r_{2}^{4}+c_{1} r_{2}^{2}+c_{0}=0
$$

where coefficients $c_{0} c_{1}, c_{2}$, and $c_{3}$, are not reproduced here for the sake of brevity. Equation (17) can be numerically solved by using the Newton-Raphson procedure. The stability of the mixed solutions can be examined by computing the eigenvalues of the corresponding Jacobain matrix of the first three equations of equation (14). The corresponding characteristic polynomial is given by

$$
\lambda^{3}+a_{2} \lambda^{2}+a_{1} \lambda+a_{0}=0
$$

where $a_{2}=2 \mu_{1}+\mu_{2}-4 s_{11} r_{1}^{2}-2 s_{12} r_{2}^{2}-s_{21} r_{1}^{2}-3 s_{22} r_{2}^{2}$, and coefficients $a_{1}$ and $a_{0}$ are not produced here for brevity. According to Hurwitz criterion, the mixed solutions are stable when $a_{2}>0, a_{1} a_{2}-a_{0}>0, a_{0}\left(a_{1} a_{2}-a_{0}\right)>0$.

The above analysis indicates that the primary resonance response of the system (1) at the first Hopf bifurcation may be periodic motion or quasi-periodic motions on a 2D torus.

\section{Primary resonance at the frequency of the second Hopf bifurcation}

At primary resonance to the second Hopf bifurcation, the frequency of external excitation is assumed to be $\delta_{2}=\Omega+\varepsilon \sigma_{2}$, where $\sigma_{2}$ is an external detuning parameter.

The first-order approximate solutions can be written in a general form as

$$
\begin{aligned}
z_{11}=r_{1} \cos \left(\delta_{1} T_{0}+\phi_{1}\right), & z_{21}=-r_{1} \sin \left(\delta_{1} T_{0}+\phi_{1}\right), \\
z_{31}=r_{2} \cos \left(\Omega T_{0}+\phi_{2}\right), & z_{41}=-r_{2} \sin \left(\Omega T_{0}+\phi_{2}\right) .
\end{aligned}
$$

where $r_{1}, r_{2}, \phi_{1}, \phi_{2}$ represent, respectively, the amplitudes and phases of the primary resonance response at the second Hopf bifurcation frequency.

The amplitudes of the primary resonance response at the second Hopf bifurcation also admits two types of solutions, either the single solutions having $r_{1}=0$ and $r_{2} \neq 0$, or mixed solutions with $r_{1} \neq 0$ and $r_{2} \neq 0$. 
For the single solutions, the so-called frequency-response equation is given by

$$
\left[\left(\mu_{2}-s_{22} r_{2}^{2}\right)^{2}+\left(\rho_{2}+s_{42} r_{2}^{2}\right)\right] r_{2}^{2}=e_{3}^{2}+e_{4}^{2}
$$

For the mixed solutions, the amplitudes of the mixed solutions are determined by two algebraic equations

$$
s_{11} r_{1}^{2}+s_{12} r_{2}^{2}-\mu_{1}=0, \quad b_{3} r_{1}^{6}+b_{2} r_{1}^{4}+b_{1} r_{1}^{2}+a_{0}=0,
$$

where coefficients, $b_{0}, b_{1}, b_{2}$ and $b_{3}$ are not reproduced here for brevity.

The stability of the single and mixed solutions can be studied in a similar procedure to that developed in Section 5 for primary resonance at the first Hopf bifurcation frequency.

\section{Illustrative examples}

Except the amplitude and frequency of the excitation, the other system parameters are identical to those as given in Section 4.2. The amplitude and frequency of the excitation will be different in order to locate primary resonances at the first and second Hopf bifurcations. Numerical simulations have shown a similar qualitative behaviour for both primary resonances at the first and second Hopf bifurcation. For brevity, only typical results of primary resonance response at the first Hopf bifurcation are given to illustrate the frequency response and an existence of two quasi-periodic solutions.

Figure 2a shows the frequency response of the system under primary resonances at the first Hopf bifurcation for $\sigma_{1}=-0.18$ (i.e. $\Omega_{0}=1.31332$ ) and $e_{0}=0.006$, with an increase of the amplitude of excitation $e_{0}$ in the region $e_{0} \in[0.001,0.04]$. Figure $2 \mathrm{~b}$ is an enlargement of the frequency response in the region $e_{0} \in[0.001,0.01]$. In these two figures, $r_{1 s}, r_{1 m}$ and $r_{2 m}$ denote the single solutions and mixed solutions of the amplitudes of primary resonance response at the first Hopf bifurcation. The stable solutions obtained from equations (15) and (17) are indicated by solid curves. The unstable periodic solutions, which result from a real eigenvalue of equation (16) crossing the imaginary axis, are 
displayed by long dot-dashed lines. The unstable periodic solutions, associated with a pair of complex eigenvalues crossing the imaginary axis which results in a Neimark-Sacker bifurcation, are represented by dashed lines. The Neimark-Sacker bifurcation of a periodic solution produces a quasiperiodic solution.

It is easy to note that both single solutions and mixed solutions exist when the amplitude of the excitation $e_{0}$ is small. When $e_{0}>0.0073$, the mixed solutions cease to exist. On the contrary, the primary resonance response admits the single solutions only when $e_{0}>0.0073$. Figure 2(b) shows that the mixed solutions are stable if they exist while single solutions are unstable till point A at $e_{0}=0.0065$, where a Neimark-Sacker bifurcations occurs. This unstable single solution will exhibit a quasi-periodic motion when doing numerical simulation on the original equation (1). For the single solutions, the branch of amplitude dashed curve B-C is unstable, as the real part of at least one eigenvalue is positive. Saddle bifurcations occur at points $\mathrm{B}$ and $\mathrm{C}$ when $e_{0}$ increases from a small value or decrease from a large value. An upward jump and downward jump occurs at points B and C. Two stable single solutions having different amplitudes coexist in the region between two points. The frequency-response curve of the system for the single solutions is very different from the one of a typical Duffing oscillator under primary resonances $[12,13]$.

Multiple solutions coexist in the region $e_{0} \in[0.001,0.0073]$, where two quasi-periodic solutions appear when doing numerical simulations to equation (1) under different sets of initial conditions. The large-amplitude quasi-periodic motion corresponds to a stable mixed solution as shown in Figure 2b, while the small-amplitude quasi-periodic motion results from an unstable single solution. Figure 3 shows the time histories and phase portraits of two quasi-periodic motions of the system with identical system parameters but under 
different sets of initial conditions. The numerical results on the coexistence of two quasiperiodic motions are in good agreement with the analytical predictions.

\section{Conclusion}

A non-resonant bifurcation of Hopf-Hopf interactions may appear in the controlled van der Pol-Duffing oscillator after the trivial equilibrium loses its stability. The presence of a periodic external excitation in the controlled oscillator at non-resonant bifurcations of codimension two can induce non-resonances, primary resonances, super- and sub-harmonic resonances, additive and difference resonances, when the frequency of the excitation and the frequencies of a double Hopf bifurcation satisfy a certain relationship.

The non-resonant and primary resonance responses of the controlled oscillator at nonresonant bifurcations of codimension two have been studied using centre manifold theorem and the method of multiple scales. It was shown that the non-resonant response of the forced oscillator may exhibit periodic motion, quasi-periodic motions on a 2D or 3D torus. The primary resonance response may exhibit single solutions or mixed solutions. The single solution may lose its stability via a saddle-node bifurcation which leads to jump phenomena or a Neimark-Sacker bifurcation which gives rise to a quasi-periodic motion. The co-existence of two quasi-periodic motions has been found using different sets of initial conditions.

\section{References}

[1] Campbell, S.A., Belair, J., 1999 Resonant co-dimension two bifurcation in the harmonic oscillator with delayed forcing, Canadian Applied Mathematics Quarterly, vol.7, pp.218-238.

[2] Maccari, A., 2001 The response of a parametrically excited van der Pol oscillator to a time delay state feedback, Nonlinear Dynamics, vol.26, pp105-119. 
[3] Xu, J., Chung, K.W., 2003 Effects of time delayed position feedback on a van der PolDuffing oscillator, Physica D, vol.180, pp.17-39.

[4] Xu, J., Yu, P., 2004, Delay-induced bifurcation in an nonautonomous system with delayed velocity feedbacks. International Journal of Bifurcation and Chaos, vol.14, pp.2777-2798.

[5] Maccari, A., 2006 Vibration control for parametrically excited Lienard systems, International Journal of Non-Linear Mechanics, vol.41, pp.146-155.

[6] Ji, J.C., Hansen, C.H., 2006 Stability and dynamics of a controlled van der Pol-Duffing oscillator, Chaos, Solitons and Fractals, vol.28(2), pp.555-570.

[7] Ji, J.C., 2006 Nonresonant Hopf bifurcations of a controlled van der Pol-Duffing oscillator, Journal of Sound and Vibration, vol.297(1-2), pp.183-199.

[8] Guckenheimer, J., Holmes, P., Nonlinear Oscillations, Dynamical Systems, and Bifurcations of Vector Fields, Springer-verlag, New York (1983).

[9] Hale, J., Theory of Functional Differential Equations, Springer, New York, 1977.

[10] Hale, J.K., Verduyn Lunel, S.M., Introduction to Functional Differential Equations, Springer, New York, 1983.

[11] Halanay, A., Differential Equations, Stability, Oscillations, Time Lags, Academic Press, New York, 1966.

[12] Nayfeh, A.H., Mook, D.T., Nonlinear Oscillations, Wiley, New York (1979).

[13] Kapitaniak T. Chaotic oscillations in mechanical systems. Manchester: Manchester University Press; 1991. 

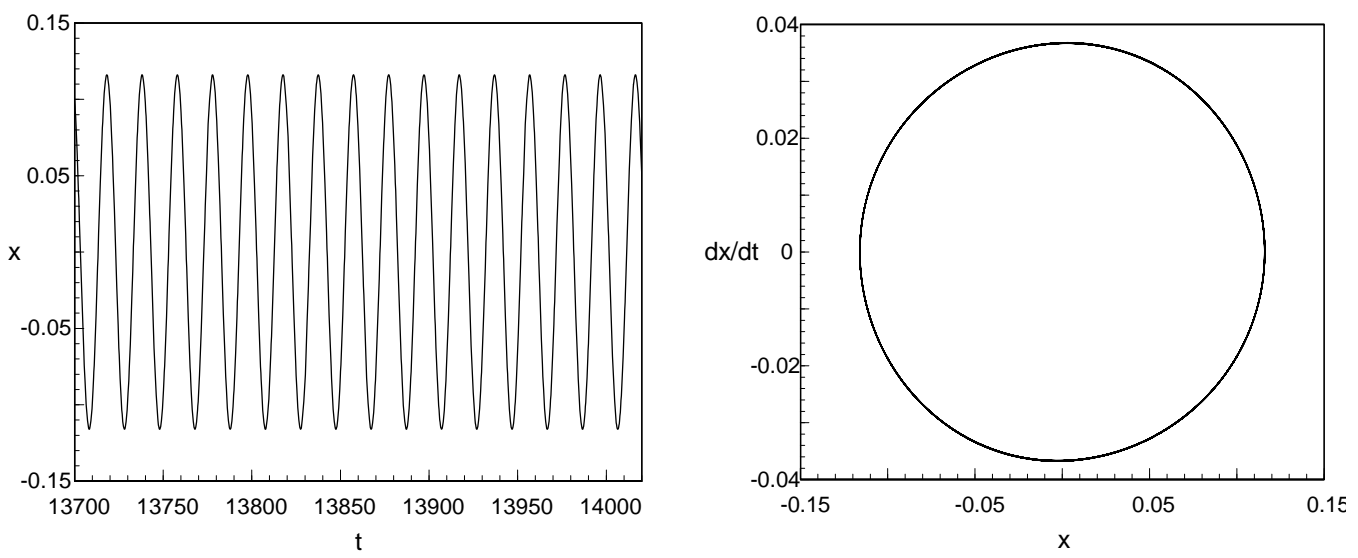

(a) Solution S1
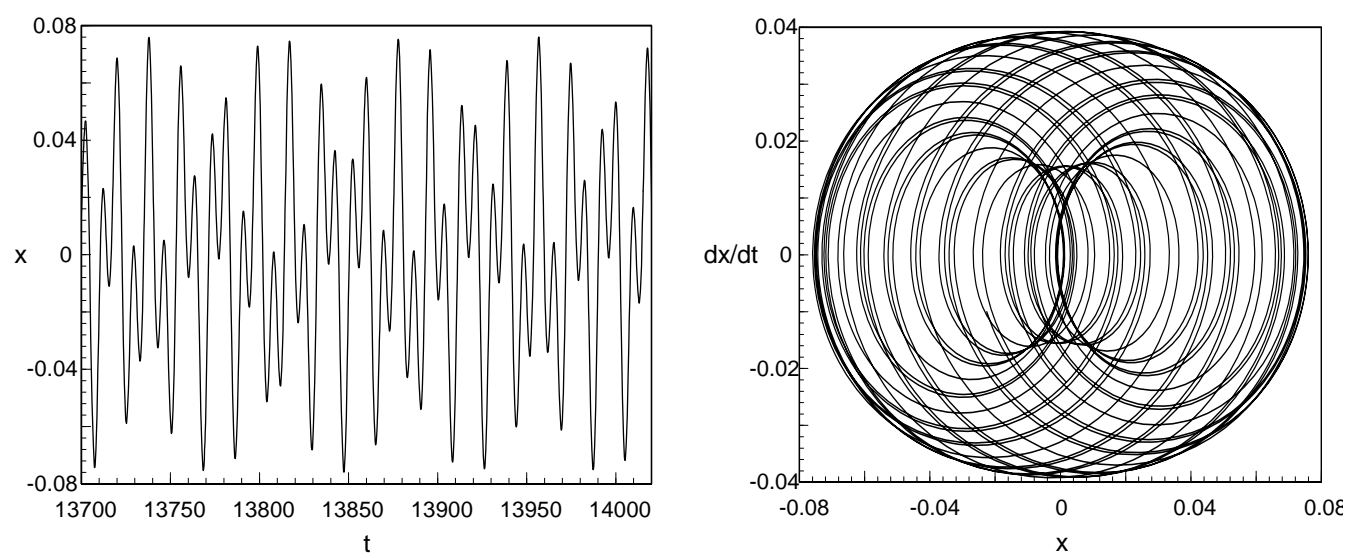

(b) Solution S2
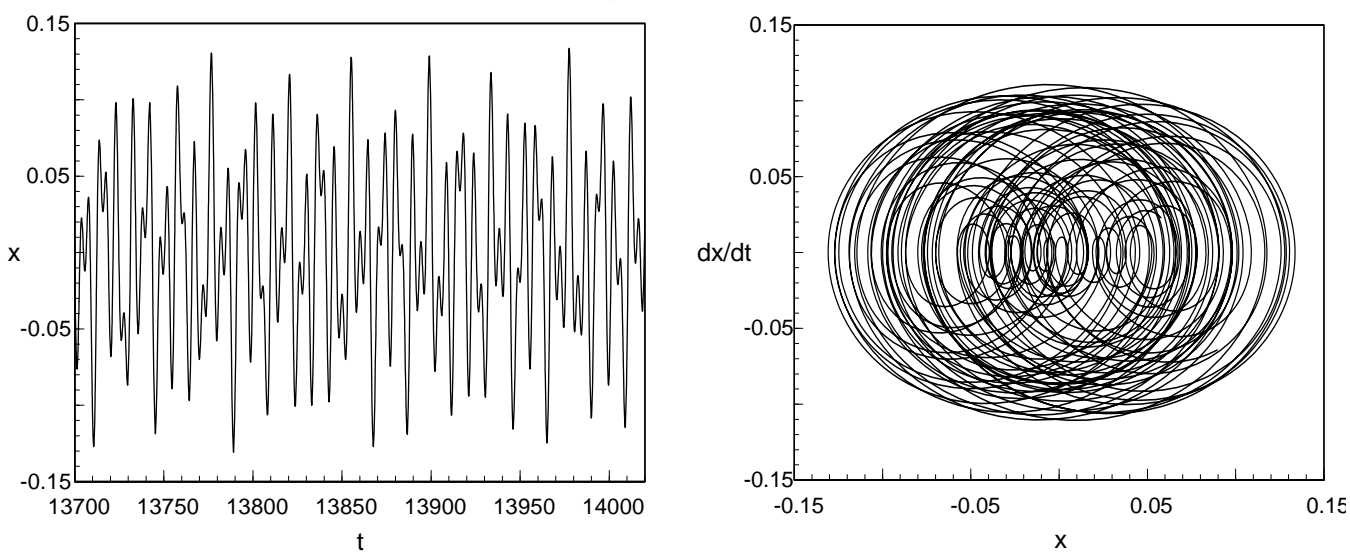

(c) Solution S4

Figure 1. Time histories and phase portraits of non-resonant response for $\Omega_{0}=0.3$; (a) a periodic solution for $\alpha_{1}=0.001, \alpha_{2}=0.1$ and $e_{0}=0.06$; (b) a 2D torus solution for $\alpha_{1}=0.001, \alpha_{2}=-0.0035$ and $e_{0}=0.04$; (c) a 3D torus solution for $\alpha_{1}=0.001$, $\alpha_{2}=0.0053$, and $e_{0}=0.016$. 

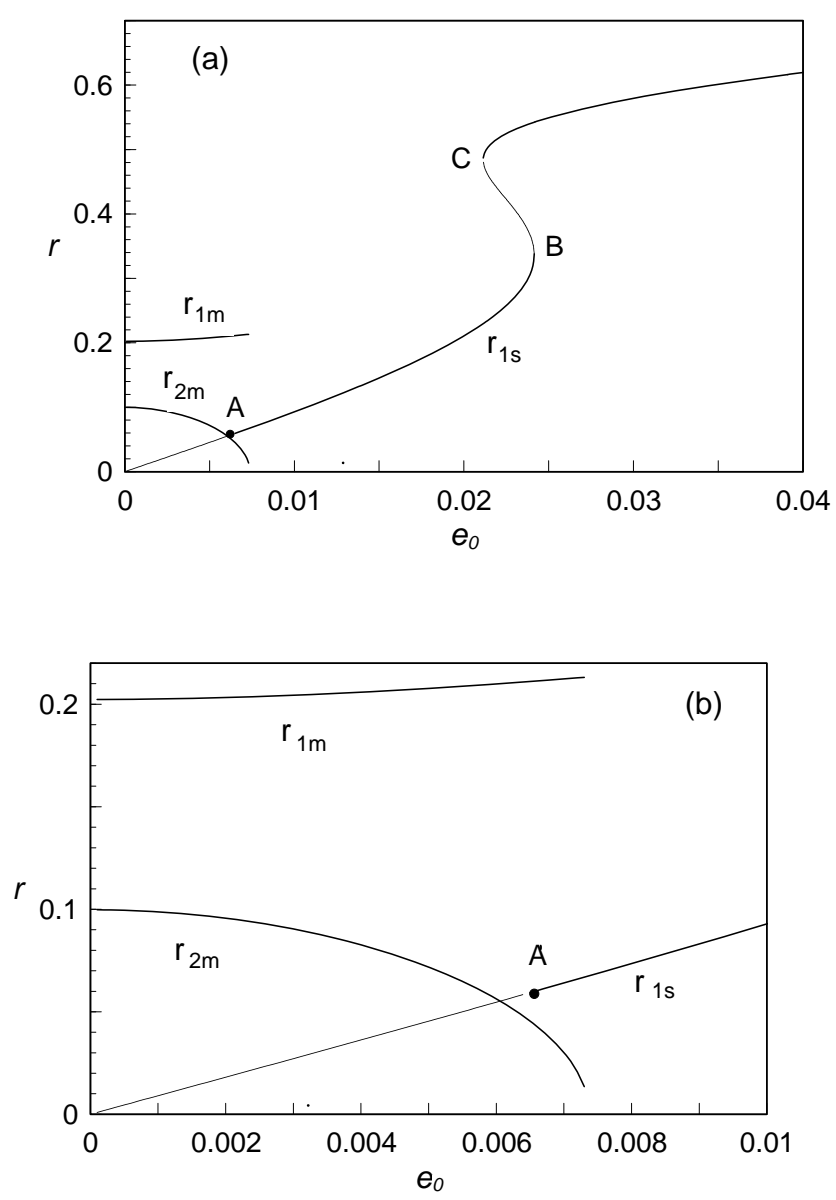

Figure 2. Frequency response of the controlled van der Pol-Duffing oscillator given by equation (1) under primary resonances at the first Hopf bifurcation. $r_{1 s}, r_{1 m}$ and $r_{2 m}$ denote the single solutions and mixed solutions to the amplitudes of the primary resonance responses. Solid curves represent stable solutions while unstable solutions are denoted by long dot-dashed lines and dashed lines. 

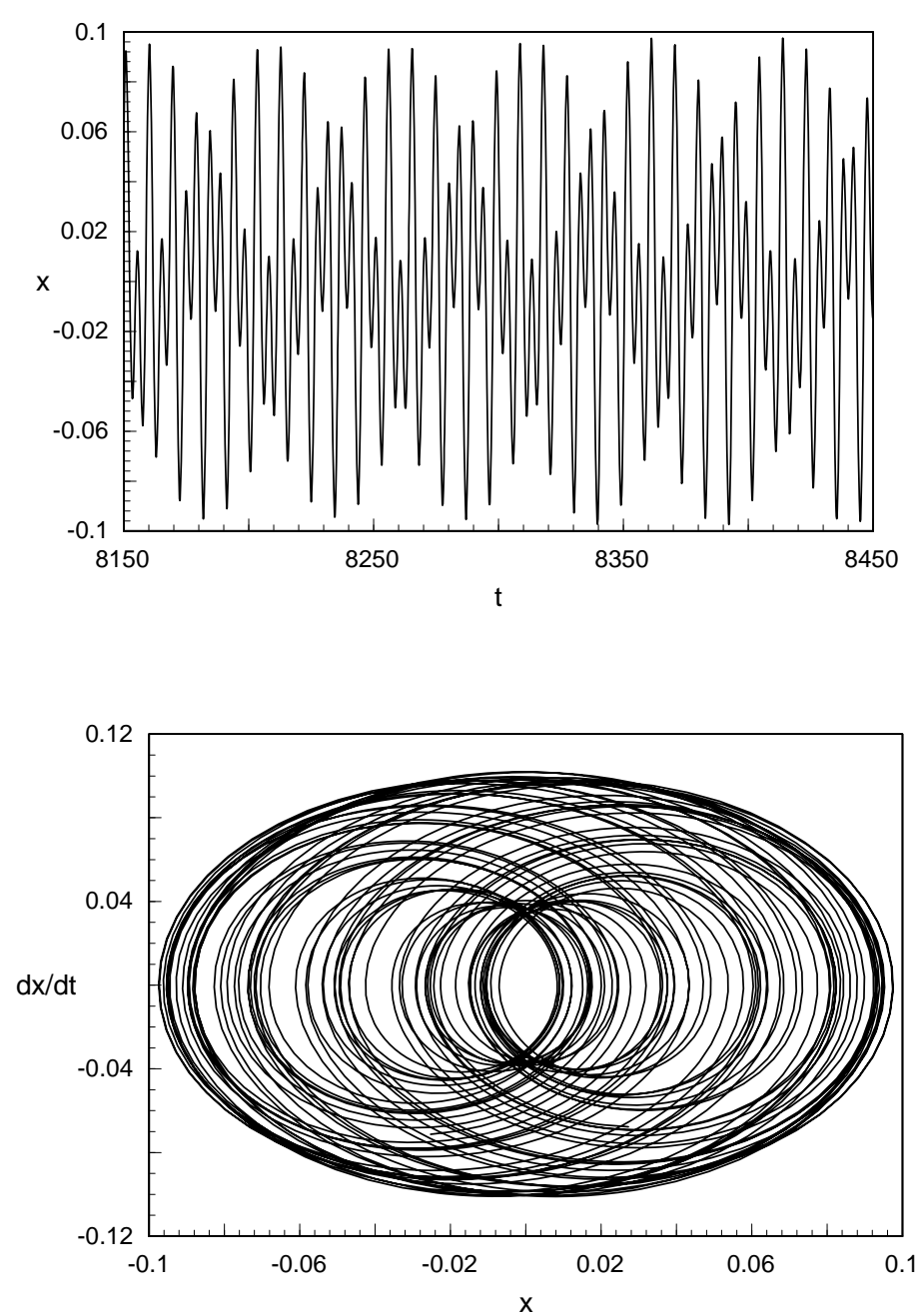

(a)

Figure 3. Time trajectories and phase portraits of two quasi-periodic solutions of the controlled system in the neighbourhood of primary resonances at the first Hopf bifurcation for $e_{0}=0.006$; (a) numerical integration starting from the initial conditions $(x, \dot{x})=(-1.1,1.0)$; (b) numerical integration starting from the initial conditions $(x, \dot{x})=(-0.05,-0.01)$. 

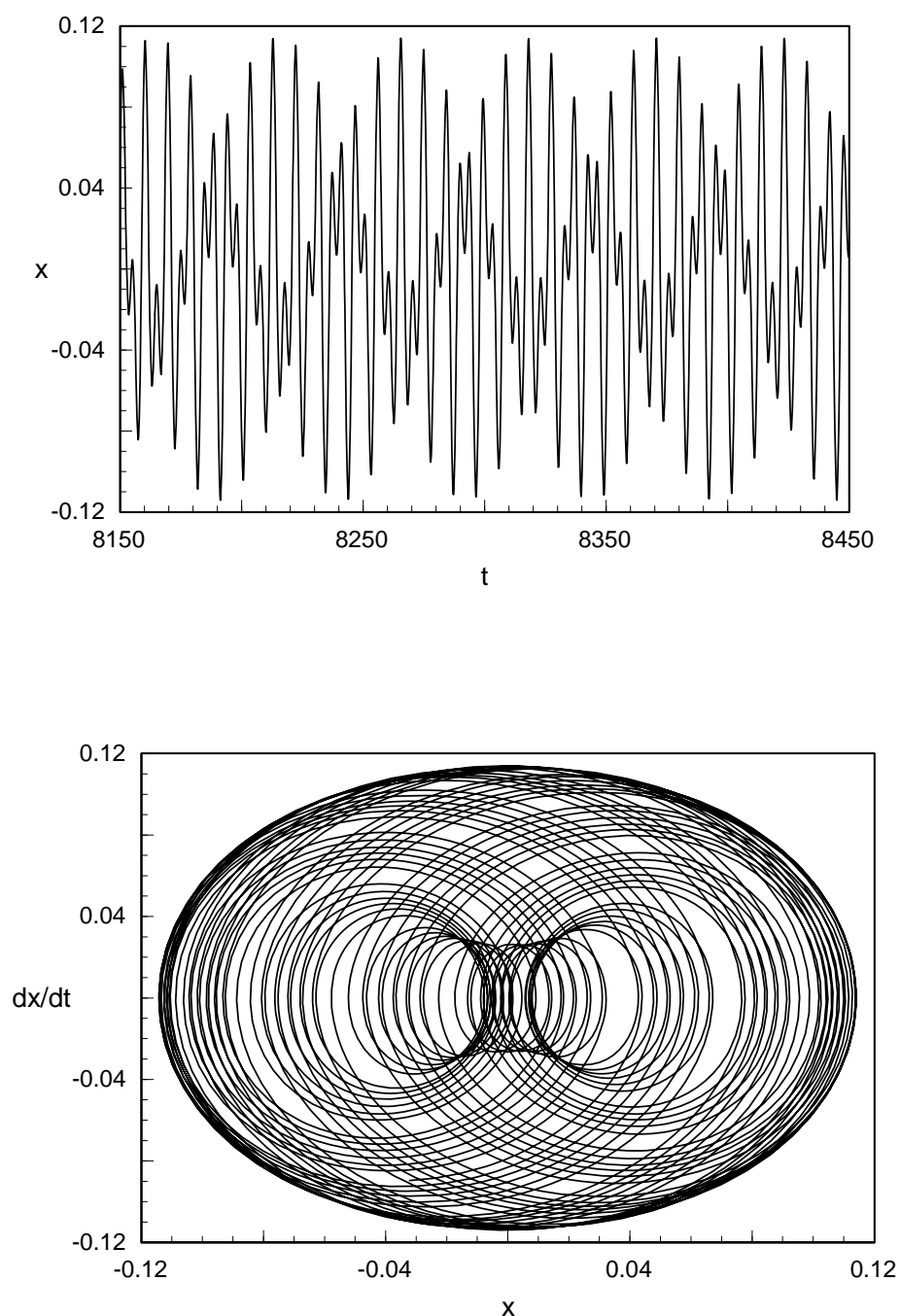

(b)

Figure 3. Time trajectories and phase portraits of two quasi-periodic solutions of the controlled system in the neighbourhood of primary resonances at the first Hopf bifurcation for $e_{0}=0.006$; (a) numerical integration starting from the initial conditions $(x, \dot{x})=(-1.1,1.0)$; (b) numerical integration starting from the initial conditions $(x, \dot{x})=(-0.05,-0.01)$. 